\title{
REFLEXIVITY, SUPPORTS AND SPECTRAL SYNTHESIS
}

\author{
A. KATAVOLOS \\ Department of Mathematics, \\ University of Athens \\ 15784 Athens \\ GREECE
}

The purpose of this talk is twofold. In the first part (sections 1-4) I will briefly describe the notions of generalised reflexivity and strong reflexivity for linear space of operators, as well as the problem of the density of the rank one subspace. The second part is devoted to a presentation of recent joint work with John Erdos and Victor Shulman [9] concerning reflexive subspaces admitting actions of masas. The perhaps surprising solution of the rank one density problem will be given, and a new "simultaneous coordinatisation" of such subspaces will be presented. This will be given in measure-theoretic terms, and so the blanket assumption of separability of all Hilbert spaces will be made (although many results, particularly in the first part, are valid generally). The results in the first part are mostly known, apart from a few exceptions (Theorem 2.2, for example); the treatment is somewhat new ${ }^{1}$.

Let me emphasise that the objects in this talk are linear spaces of operators acting on a Hilbert space. The basic notions we are interested in (reflexivity, rank one operators etc.) are not invariant under isomorphism, but only under spatial isomorphism. Thus the notion of equivalence will be unitary equivalence, not some more general notion of isomorphism.

Why linear spaces and not (unital) algebras? As we will see, many results in operator algebra theory do not depend essentially on multiplication of operators; furthermore, in some problems concerning operator algebras,

\footnotetext{
${ }^{1}$ This approach is based on seminar notes which were privately circulated in 1994 under the title "Killers and preduals".
} 
linear spaces which are not algebras (such as annihilators) appear quite naturally. Thus in some instances the extra structure of multiplication (or the ${ }^{*}$-operation) may obscure the issue.

\section{Reflexivity}

\subsection{BASIC CONCEPTS}

The concept of reflexivity for algebras of operators (and its dual concept, reflexivity for lattices of subspaces) was formally introduced by Halmos [12], although particular instances of reflexive algebras were considered earlier (see [16], [25]).

Recall that a unital algebra $\mathcal{A}$ of operators on a Hilbert space $\mathcal{H}$ is said to be reflexive if any $T \in \mathcal{B}(\mathcal{H})$ which leaves invariant all (closed) $\mathcal{A}$ invariant subspaces (that is, all elements of the lattice $\operatorname{Lat}(\mathcal{A})$ of $\mathcal{A}$-invariant subspaces) is itself in $\mathcal{A}$. A moment's reflection shows that this happens if and only if $T x$ is in the closure of $\mathcal{A} x$ for all $x \in \mathcal{H}$. It is the latter property which proves to be fruitful when $\mathcal{A}$ is a subspace of operators which need not be an algebra or contain the identity; in fact the property makes sense even when the operators map the space $\mathcal{H}$ to some (perhaps different) Hilbert space $\mathcal{K}$. This leads to the following

Definition 1.1 (Loginov-Shulman [21], Erdos [8])

(i) The reflexive cover $\operatorname{Ref}(\mathcal{S})$ of a subset $\mathcal{S} \subseteq \mathcal{B}(\mathcal{H}, \mathcal{K})$ is the set of all $B \in \mathcal{B}(\mathcal{H}, \mathcal{K})$ such that

$$
B x \in \overline{[\mathcal{S} x]} \quad \forall x \in \mathcal{H} .
$$

(ii) A subset $\mathcal{S} \subseteq \mathcal{B}(\mathcal{H}, \mathcal{K})$ is said to be reflexive if $\mathcal{S}=\operatorname{Ref}(\mathcal{S})$.

Clearly $\operatorname{Ref}(\mathcal{S})$ is a weak operator closed subspace of $\mathcal{B}(\mathcal{H}, \mathcal{K})$ containing $\mathcal{S}$. The above remarks show that, when $\mathcal{A} \subseteq \mathcal{B}(\mathcal{H})$ is a unital algebra, then $\operatorname{Ref}(\mathcal{A})=\operatorname{Alg} \operatorname{Lat}(\mathcal{A})$, the unital algebra of all operators leaving invariant all $\mathcal{A}$-invariant subspaces. In general, (for $\mathcal{H}=\mathcal{K}$ ) one has $\operatorname{Ref}(\mathcal{S}) \subseteq \operatorname{Alg} \operatorname{Lat}(\mathcal{S})$, and the inclusion may be strict:

Example 1 Let $\mathcal{S} \subseteq \mathcal{B}\left(\mathbb{C}^{2}\right)$ be the algebra of all strictly upper-triangular operators. Then Lat $\mathcal{S}$ is the nest $\left\{\{0\},\left[e_{1}\right], \mathbb{C}^{2}\right\}$. Here $I \in \operatorname{Alg} \operatorname{Lat}(\mathcal{S})$ but $I \notin \operatorname{Ref}(\mathcal{S})$ because $\mathcal{S} e_{1}=\{0\}$. In this example

$$
\mathcal{S}=\operatorname{Ref}(\mathcal{S})=\left(\begin{array}{c}
* \\
\end{array}\right) \quad \operatorname{Alg} \operatorname{Lat}(\mathcal{S})=\left(\begin{array}{cc}
* & * \\
& *
\end{array}\right)
$$


Example 2 Let $\mathcal{S} \subseteq \mathcal{B}\left(\mathbb{C}^{3}\right)$ be the unital subspace generated by the matrix units $E_{i i}(i=1,2,3), E_{12}$ and $E_{23}$. Here

$$
\mathcal{S}=\left(\begin{array}{ccc}
* & * & \\
& * & * \\
& & *
\end{array}\right), \quad \operatorname{Alg} \operatorname{Lat}(\mathcal{S})=\left(\begin{array}{ccc}
* & * & * \\
& * & * \\
& & *
\end{array}\right) .
$$

This is not an algebra because $\left(E_{12}+E_{23}\right)^{2}=E_{13} \notin \mathcal{S}$. Note that Lat $\mathcal{S}$ is the nest $\left\{\{0\},\left[e_{1}\right],\left[e_{1}, e_{2}\right], \mathbb{C}^{3}\right\}$, so $E_{13} \in \operatorname{Alg} \operatorname{Lat}(\mathcal{S})$. But $E_{13} \notin \operatorname{Ref}(\mathcal{S})$ because $E_{13} e_{3}=e_{1}$ while $\mathcal{S} e_{3} \perp e_{1}$.

Note that in both examples $\mathcal{S}$ is a bimodule over the masa (:maximal abelian selfadjoint algebra) of all diagonal matrices.

Even in the study of reflexive algebras, reflexive subspaces which are not algebras arise quite naturally: as we shall see (Theorem 2.1), the annihilator of the rank-one subalgebra of a reflexive algebra is a reflexive subspace, but it is very seldom an algebra.

Given any set $\mathcal{S} \subseteq \mathcal{B}(\mathcal{H})$ of operators, the smallest reflexive (unital) algebra containing $\mathcal{S}$ may be determined in two steps, by first finding the lattice $\mathcal{L}=\operatorname{Lat}(\mathcal{S})$ of all closed $\mathcal{S}$-invariant subspaces and then finding the unital algebra $\operatorname{Alg} \mathcal{L}$ of all operators which leave all members of $\mathcal{L}$ invariant:

$$
\mathcal{S} \rightarrow \operatorname{Lat}(\mathcal{S}) \rightarrow \operatorname{Alg} \operatorname{Lat}(\mathcal{S})
$$

Erdos [8] defines an analogous two-step process for finding the reflexive cover of a set $\mathcal{S} \subseteq \mathcal{B}(\mathcal{H}, \mathcal{K})$ : one first finds the map $\phi_{\mathcal{S}}$ determined by $\mathcal{S}$ which associates to any (closed) subspace $P$ of $\mathcal{H}$ the cyclic subspace $\overline{\mathcal{S}(P)}$. Then $\operatorname{Ref}(\mathcal{S})$ equals $O p \phi_{\mathcal{S}}$, the set of all operators $T$ that 'respect' this map in the sense that $T(P) \subseteq \phi_{\mathcal{S}}(P)$ for all $P$ :

$$
\mathcal{S} \rightarrow \phi_{\mathcal{S}} \rightarrow O p \phi_{\mathcal{S}}=\operatorname{Ref}(\mathcal{S}) .
$$

Any subspace lattice $\mathcal{L}$ determines a map $\phi_{\mathcal{L}}$ by

$$
\phi_{\mathcal{L}}(P)=\wedge\{L \in \mathcal{L}: L \supseteq P\} .
$$

Note that $\left.\phi_{\mathcal{L}}\right|_{\mathcal{L}}$ is the identity map. Then $O p \phi_{\mathcal{L}}=\operatorname{Alg}(\mathcal{L})$. But other maps are useful, even in the unital algebra case: if $\phi_{\mathcal{L}}^{\vee}$ is defined by

$$
\phi_{\mathcal{L}}^{\vee}(P)=\vee\{L \in \mathcal{L}: L \underline{\perp} P\}
$$


then it turns out that $O p \phi_{\mathcal{L}}^{\vee}$ is precisely the annihilator of the rank one subspace of $\operatorname{Alg}(\mathcal{L})$. Note that, for $P \in \mathcal{L}, \phi_{\mathcal{L}}^{\vee}(P)$ is the 'generalised predecessor' $P_{-}$of $P$ introduced by Longstaff [22].

A reflexive (unital) subalgebra $\mathcal{A}$ of $\mathcal{B}(\mathcal{H})$ is determined by the set Lat $\mathcal{A}$ of its invariant subspaces. In the same way, a reflexive subspace $\mathcal{S} \subseteq \mathcal{B}(\mathcal{H}, \mathcal{K})$ is determined by its map $\phi_{\mathcal{S}}$; its invariant lattice is in general not large enough to determine it.

Example Consider the ultraweakly closed subspace $\mathcal{S} \subseteq \mathcal{B}\left(\ell^{2}\right)$ generated by the matrix units $\left\{E_{n, n}: n \in \mathbb{N}\right\},\left\{E_{n+1, n}: n \in \mathbb{N}\right\}$ and $\left\{E_{1, n}: n \in \mathbb{N}\right\}$.

$$
\mathcal{S}=\left(\begin{array}{ccccc}
* & * & * & * & \cdots \\
* & * & & & \\
& * & * & & \\
& & * & * & \\
& & & \ddots & \ddots
\end{array}\right)
$$

We will show below (section 6) that any subspace which is the ultraweakly closed span of a set of matrix units is necessarily reflexive. However it can be verified that in this case Lat $\mathcal{S}$ is trivial and so cannot determine $\mathcal{S}$.

\section{2. 'CLOSURES'}

The reflexive cover of a set of operators can be thought of as its "onepoint closure". Of course Ref is not a closure operator in the topological sense. However, in some important cases, the reflexive cover of a subspace coincides with its closure in the weak operator topology (WOT) or even the ultraweak topology $\left(\mathrm{w}^{*}\right)$.

In fact, the von Neumann bicommutant theorem says that if $\mathcal{A} \subseteq \mathcal{B}(\mathcal{H})$ is a unital *algebra, then $\operatorname{Ref}(\mathcal{A})=\mathrm{w}^{*}$-cl $(\mathcal{A})$ (because $\operatorname{Ref}(\mathcal{A})=\mathcal{A}^{\prime \prime}$ for unital *-algebras). Of course this fails miserably in the non-selfadjoint case: for example if

$$
\mathcal{A}=\left\{\left(\begin{array}{ll}
a & b \\
0 & a
\end{array}\right): a, b \in \mathbb{C}\right\}
$$

then $\operatorname{Ref}(\mathcal{A})$ is the set of all upper-triangular matrices.

These observations motivate the general theme of this talk:

Theme: When do the equalities

$$
\operatorname{Ref}(\mathcal{S})=\overline{\mathcal{S}}^{W O T}
$$


or

$$
\operatorname{Ref}(\mathcal{S})=\overline{\mathcal{S}}^{w *}
$$

hold?

We will see below (section 6 ) that if $\mathcal{S} \subseteq \mathcal{B}(\mathcal{H})$ is the linear span of a set of matrix units, then (2) holds (hence (1) also holds). Note that in this case $\mathcal{S}$ is a bimodule over the discrete masa generated by the diagonal matrix units.

More generally, if $\mathcal{S}$ is a bimodule over a masa $\mathcal{A}$ (that is, $\mathcal{A S} \subseteq \mathcal{S}$ and $\mathcal{S A} \subseteq \mathcal{S})$ and is the linear span of a set of rank one operators, then (1) holds (theorem 5.2) but (2) may fail (section 10).

\section{Reflexivity and rank one operators}

A crucial observation is that reflexive subspaces can be characterised in terms of rank one operators ${ }^{2}$. Indeed,

$$
\begin{aligned}
\operatorname{Ref}(\mathcal{S}) & =\{T \in \mathcal{B}(\mathcal{H}, \mathcal{K}):\langle\mathcal{S} x, y\rangle=0 \Rightarrow\langle T x, y\rangle=0\} \\
& =\left\{T \in \mathcal{B}(\mathcal{H}, \mathcal{K}): x \otimes y^{*} \perp \mathcal{S} \Rightarrow x \otimes y^{*} \perp T\right\} \\
& =\left(\mathcal{R}_{1}\left({ }^{\perp} \mathcal{S}\right)\right)^{\perp}
\end{aligned}
$$

where $\mathcal{R}_{1}(\mathcal{T})$ denotes the rank one subspace of $\mathcal{T}$ (the linear span of the rank one operators in $\mathcal{T}$ ) and $x \otimes y^{*}(\eta)=\langle\eta, y\rangle$.

Thus reflexive spaces are (post-) annihilators of sets of rank ones. The converse also holds. Thus

Theorem 2.1 A set $\mathcal{S} \subseteq \mathcal{B}(\mathcal{H}, \mathcal{K})$ is reflexive if and only if it is of the form $\mathcal{S}=\mathcal{R}^{\perp}$, for some set $\mathcal{R} \subseteq \mathcal{B}(\mathcal{K}, \mathcal{H})$ of rank one operators.

Proof Let $\mathcal{S}=\mathcal{R}^{\perp}$. Suppose that $T x \in \overline{\mathcal{S} x}$ for all $x \in H$. Then for each $x \otimes y^{*} \in \mathcal{R}$, we have $x \otimes y^{*} \perp \mathcal{S}$, i.e. $\langle\mathcal{S} x, y\rangle=0$ and hence $\langle T x, y\rangle=0$. Thus $T \in \mathcal{R}^{\perp}=\mathcal{S}$.

An interesting application of this concerns "approximate decomposability" of operators with respect to reflexive subspaces:

\footnotetext{
${ }^{2}$ This is due to Larson [18] in the case of unital algebras, and to Kraus-Larson [15] and Erdos [8] in the general case. Theorem 2.1 is from [8], Theorem 9.2.
} 
Theorem 2.2 Let $\mathcal{S} \subseteq \mathcal{B}(\mathcal{H}, \mathcal{K})$ be reflexive, and let $\mathcal{T}$ be the reflexive subspace $\left(\mathcal{R}_{1}(\mathcal{S})\right)^{\perp}$. Then every Hilbert-Schmidt operator $T$ on $\mathcal{H}$ decomposes as a sum $T_{1}+T_{2}$ of two Hilbert-Schmidt operators such that $T_{1} \in \mathcal{S}$ and $T_{2}^{*} \in \mathcal{T}$. This decomposition is unique if and only if every Hilbert-Schmidt operator in $\mathcal{S}$ is in the $\mathcal{C}_{2}$-closure of $\mathcal{R}_{1}(\mathcal{S})$. In particular, the sum $\mathcal{S}+\mathcal{T}^{*}$ is ultraweakly dense in $\mathcal{B}(\mathcal{H}, \mathcal{K})$.

Proof Let $\mathcal{S}_{2}=\mathcal{C}_{2} \cap S, \mathcal{T}_{2}=\mathcal{C}_{2} \cap \mathcal{T}$ and let $A \in \mathcal{C}_{2}$ be orthogonal to $\mathcal{T}_{2}$ in the Hilbert space $\mathcal{C}_{2}$. Thus $\operatorname{tr}\left(A^{*} T\right)=0$ for all $T \in \mathcal{C}_{2}$ that annihilate $\mathcal{R}_{1}(\mathcal{S})$, and hence $A^{*} \in{\overline{\mathcal{R}_{1}(\mathcal{S})}}^{2}$ (the closure of $\mathcal{R}_{1}(\mathcal{S})$ in the Hilbert-Schmidt norm). It follows easily that the orthogonal complement of $\mathcal{T}_{2}$ is precisely $\overline{\mathcal{R}_{1}\left(\mathcal{S}^{*}\right)}$. Thus

$$
\mathcal{C}_{2}=\mathcal{T}_{2}+\mathcal{T}_{2}^{\perp} \subseteq \mathcal{T}_{2}+\mathcal{S}_{2}^{*} \subseteq \mathcal{C}_{2}
$$

hence equality holds. The sum $\mathcal{T}_{2}+\mathcal{S}_{2}^{*}$ is direct if and only if ${\overline{\mathcal{R}_{1}(\mathcal{S})}}^{2}=$ $\mathcal{S}_{2}$.

In the case of a unital algebra, this result was obtained jointly with M. Papadakis [24]. It allows a quick proof of various decomposability (sometimes called *-density) results, the oldest of which is attributed to Kadison and Singer [16] (see also [1], [3], [11]): If $\mathcal{A} \subseteq \mathcal{B}(\mathcal{H})$ is a nest algebra, then $\mathcal{A}+\mathcal{A}^{*}$ is ultraweakly dense in $\mathcal{B}(\mathcal{H})$. Indeed, in this case one easily verifies that $\left(\mathcal{R}_{1}(\mathcal{A})\right)^{\perp} \subseteq \mathcal{A}$. Thus the theorem may be considered as a generalisation of the decomposability of a matrix in its upper triangular and strictly lower triangular parts.

Remark 2.1 Theorem 2.1 leads to the following reformulation of our basic theme for a subspace $\mathcal{S} \subseteq \mathcal{B}(\mathcal{H}, \mathcal{K})$ :

$$
\operatorname{Ref}(\mathcal{S})=\overline{\mathcal{S}}^{w *} \Leftrightarrow{\overline{\mathcal{R}_{1}\left({ }^{\perp} \mathcal{S}\right)}}^{1}={ }^{\perp} \mathcal{S}
$$

and

$$
\operatorname{Ref}(\mathcal{S})=\overline{\mathcal{S}}^{W O T} \Leftrightarrow{\overline{\mathcal{R}_{1}\left({ }^{\perp} \mathcal{S}\right)}}^{1} \supseteq \mathcal{F}\left({ }^{\perp} \mathcal{S}\right)
$$

(here $\mathcal{F}\left({ }^{\perp} \mathcal{S}\right)$ stands for the finite rank operators in ${ }^{\perp} \mathcal{S}$.) That is, the reflexive cover of a subspace $\mathcal{S}$ coincides with its ultraweak (resp. weak operator) closure if and only if every operator (resp. every finite rank operator) in its pre-annihilator may be approximated, in trace norm, by sums of rank one operators annihilating $\mathcal{S}$.

This follows from (3) using duality theory, since $\overline{\mathcal{S}}^{W O T}=\left(\mathcal{F}\left({ }^{\perp} \mathcal{S}\right)\right)^{\perp}$ and $\overline{\mathcal{S}}^{w *}=\left({ }^{\perp} \mathcal{S}\right)^{\perp}$. 


\section{Strong reflexivity}

While there are always rank one operators annihilating a (proper) reflexive subspace, there may well be no rank one operators contained in it (example 3.1) or even no compact operators at all (example: the multiplication algebra of a non-atomic masa).

Example 3.1 Let $\mathcal{A}=\operatorname{Alg}(\mathcal{L})$ where $\mathcal{L}$ is a double triangle (that is, $\mathcal{L}$ consists of three mutually disjoint closed subspaces such that the closed span of each pair is the whole space). Then $\mathcal{A}$ contains no rank one operators.

By contrast, the annihilator of a reflexive subspace is itself a reflexive subspace containing plenty of rank one operators; in fact, it is the reflexive cover of its rank one subspace (see Proposition 3.2 below). This leads to the following

Definition 3.1 A subset $\mathcal{S} \subseteq \mathcal{B}(\mathcal{H}, \mathcal{K})$ is said to be strongly reflexive if $\mathcal{S}=\operatorname{Ref}(\mathcal{R})$ for some set $\mathcal{R}$ of rank one operators.

Then of course we can take $\mathcal{R}=\mathcal{R}_{1}(\mathcal{S})$.

The original definition of strong reflexivity generalised the notion of complete distributivity from subspace lattices to subspace maps. The equivalence of this definition to the one given above is due to John Erdos [8]. Longstaff [22] introduced this notion for unital algebras and showed that if $\mathcal{L}$ is a subspace lattice which is completely distributive, then $\mathcal{L}=\operatorname{Lat}(\mathcal{R})$ and so $\operatorname{Alg}(\mathcal{L})$ is strongly reflexive, but the converse may fail.

The prototypes of strongly reflexive algebras are nest algebras [25] and algebras whose lattices are Boolean and atomic [12]. These lattices were shown to be reflexive using the particularly simple geometric characterisation of rank one operators that leave them invariant.

But strongly reflexive subspaces are important from a different point of view, which has so far received little attention: they arise quite naturally in duality theory.

Lemma 3.1 A reflexive subspace $\mathcal{S} \subseteq \mathcal{B}(\mathcal{H}, \mathcal{K})$ is strongly reflexive if and only if any rank one which kills $\mathcal{R}_{1}(\mathcal{S})$ in fact kills all of $\mathcal{S}$.

Proof Let $\mathcal{R}=\mathcal{R}_{1}(\mathcal{S})$. We have remarked above (see (3))that $x \otimes y^{*} \perp \mathcal{R}$ if and only if $x \otimes y^{*} \perp \operatorname{Ref}(\mathcal{R})$. Thus if $\mathcal{S}=\operatorname{Ref}(\mathcal{R})$ then any rank one 
operator killing $\mathcal{R}$ also kills $\mathcal{S}$. Conversely if this latter property holds then $\mathcal{S}=\operatorname{Ref}(\mathcal{S})=\operatorname{Ref}(\mathcal{R})$

The following characterisation is essentially in [8], Theorem 9.4.

Proposition 3.2 A subspace $\mathcal{S} \subseteq \mathcal{B}(\mathcal{H}, \mathcal{K})$ is strongly reflexive if and only if $\mathcal{S}=\mathcal{R}^{\perp}$ where $\mathcal{R} \in \mathcal{B}(\mathcal{K}, \mathcal{H})$ is the set of all rank one operators in a reflexive subspace.

Proof From (3),

$$
\operatorname{Ref}\left(\mathcal{R}_{o}\right)=\left(\mathcal{R}_{1}\left({ }^{\perp} \mathcal{R}_{o}\right)\right)^{\perp} .
$$

But $\mathcal{R}_{1}\left({ }^{\perp} \mathcal{R}_{o}\right)=\mathcal{R}_{1}\left(\mathcal{R}_{o}^{\perp}\right)$ and $\mathcal{R}_{o}^{\perp}$ is a reflexive subspace.

\section{Density of the rank one subspace}

In 1968, Erdos [7] showed that in a nest algebra $\mathcal{A}$, the rank one subspace $\mathcal{R}_{1}(\mathcal{A})$ generates $\mathcal{A}$ not only as a reflexive algebra, but in fact as a $\mathrm{w}^{*}$-closed algebra. This result generated a lot of interest in the general question,

When is the rank one subspace $\mathcal{R}_{1}(\mathcal{S})$ of a reflexive space $\mathcal{S}$ dense in $\mathcal{S}$ with respect to the ultraweak, or at least the weak operator topology?

It is clear that a necessary condition is $\operatorname{Ref}\left(\mathcal{R}_{1}(\mathcal{S})\right)=\mathcal{S}$, that is, strong reflexivity of $\mathcal{S}$.

For CSL algebras, that is, reflexive algebras containing a masa, it was proved in 1983 that this condition was also sufficient for density in the ultraweak topology [20]. But the question remained open for other strongly reflexive algebras, and in particular for the important class of algebras with atomic Boolean invariant lattices. In 1991 it was realised that the answer is as negative as it could be: there exists an (abelian) algebra whose lattice is atomic Boolean, with one-dimensional atoms, in which the identity operator cannot be approximated by sums of rank one operators at two points of the space simultaneously (let alone in the weak operator topology). This is shown in the Addendum of [2] using the main example of [19] (see also J. Erdos' article in these Proceedings).

In all these results, the behaviour of strongly reflexive unital algebras with respect to rank one density in the ultraweak topology is the same as in the weak operator topology. Surprisingly, as we shall see, this is no longer true for strongly reflexive subspaces. 
The rank one density problem is equivalent to the questions:

Theme': If $\mathcal{R}$ is a set of rank one operators,

$$
\begin{aligned}
\operatorname{Ref}(\mathcal{R}) & \stackrel{?}{=} \overline{[\mathcal{R}]}^{W O T} \\
\operatorname{Ref}(\mathcal{R}) & \stackrel{?}{=} \overline{[\mathcal{R}]}^{w *}
\end{aligned}
$$

Equivalently, is $\overline{[\mathcal{R}}^{W O T}$ (resp. $\overline{[\mathcal{R}}^{w *}$ ) a reflexive subspace?

In view of Remark 2.1, these questions may be reformulated as follows

Proposition 4.1 If $\mathcal{R}$ is a set of rank one operators,

$$
\operatorname{Ref}(\mathcal{R})=\overline{[\mathcal{R}}^{\text {WOT }} \Leftrightarrow \mathcal{F}\left(\mathcal{R}^{\perp}\right) \subseteq{\overline{\mathcal{R}_{1}\left(\mathcal{R}^{\perp}\right)}}^{1}
$$

and

$$
\operatorname{Ref}(\mathcal{R})=\overline{[\mathcal{R}}^{w *} \Leftrightarrow{ }^{\perp} \mathcal{R}=\mathcal{C}_{1} \cap \mathcal{R}^{\perp}={\overline{\mathcal{R}_{1}\left(\mathcal{R}^{\perp}\right)}}^{1}
$$

Note that $\mathcal{R}^{\perp}$ (unlike ${ }^{\perp} \mathcal{S}$ as in the general case) is a reflexive subspace.

This brings us to the study of approximation of finite rank and trace class operators by sums of rank one operators.

\section{Finite rank operators in masa bimodules}

Let $\mathcal{S} \subseteq \mathcal{B}(\mathcal{H}, \mathcal{K})$ be a (perhaps reflexive) subspace. Is every finite rank operator of $\mathcal{S}$ a (finite) sum of rank one operators in $\mathcal{S}$ ?

In general, the answer is no: in Example 3.1, $\operatorname{Alg} \mathcal{L}$ never contains rank one operators, whereas (for a suitable ${ }^{3}$ double triangle $\mathcal{L}$ ) it may contain plenty of rank two operators.

Such an extreme pathology cannot happen in norm closed masa bimodules: if such an object has finite rank operators, then it also has rank one operators (however, it may contain Hilbert-Schmidt operators and no rank ones!). For CSL algebras, this was shown in [13].

In the sequel we will focus attention to masa bimodules, that is, subspaces $\mathcal{M} \subseteq \mathcal{B}(\mathcal{H}, \mathcal{K})$ admitting actions of two masas $\mathcal{A} \subseteq \mathcal{B}(\mathcal{H})$ and $\mathcal{B} \subseteq \mathcal{B}(\mathcal{K})$ in

\footnotetext{
${ }^{3}$ There exist double triangles $\mathcal{L}$ where sums of rank two operators are WOT dense in $\operatorname{Alg} \mathcal{L}$, but also others whose algebras contain no finite rank operators (see [17]).
} 
the sense that $\mathcal{B M A} \subseteq \mathcal{M}$. These objects have proved to be particularly amenable to detailed analysis.

However, even in this setting the answer to the above question is negative:

Example 5.1 (Hopenwasser-Moore [13]) There exists a (strongly) reflexive space $\mathcal{S} \subseteq \mathcal{B}\left(\ell^{2}\right)$ which is a bimodule over a totally atomic masa, and a rank two operator $T \in \mathcal{S}$ which is not a (finite) sum of rank one operators in $\mathcal{S}$ (although $\overline{\mathcal{R}}_{1}(\mathcal{S}){ }^{w *}=\mathcal{S}$ ).

Proof Consider the space $\mathcal{S}$ of all operators on $\ell^{2}$ which have zero diagonal. This is a reflexive space (it equals $\mathcal{R}^{\perp}$, where $\mathcal{R}=\left\{E_{n n}: n \in \mathbb{N}\right\}$; that it is strongly reflexive follows from Proposition 6.2 below), and a bimodule over the (discrete) masa of diagonal operators. If $x=\left(\frac{1}{n}\right)$ and $y=\left(\frac{1}{n^{2}}\right)$, one shows that the rank two operator $T=x \otimes y^{*}-y \otimes x^{*}$ cannot be written as a finite sum of rank one operators with zero diagonal. It can, of course, be approximated by such sums, even in trace norm: just let $T_{n}=P_{n} T$, where $P_{n}$ is the projection onto $\left[e_{1}, \ldots e_{n}\right]$.

Nevertheless, Davidson [6] proves that every finite rank operator in a CSL algebra is in the norm closure of the rank one subspace. In order to apply the duality methods of the previous sections, we need to extend this beyond algebras and also beyond norm closures.

Theorem 5.1 ([9]) If $\mathcal{S}$ is a norm closed masa bimodule, every finite rank operator $T$ in $\mathcal{S}$ can be approximated, even in trace-norm, by sums of rank one operators in $\mathcal{S}$.

Proof (Sketch) Suppose for simplicity that $\operatorname{rank} T=2$. Look at all compressions $F T E$ (where $F, E$ are projections in the masas) whose rank is at most 1 . If these compressions 'fill out' $T$, we are done. If not, we can find a compression $F_{o} T E_{o}$ every sub-compression of which has rank 2 or 0 . Now use the fact that the rank of $F_{o} T E_{o}$ is finite to show that no rank one operator in $\mathcal{B}\left(E_{o} \mathcal{H}, F_{o} \mathcal{K}\right)$ can annihilate $F_{o} T E_{o}$. This means that $\operatorname{Ref}\left(F_{o} \mathcal{S} E_{o}\right)=\mathcal{B}\left(E_{o} \mathcal{H}, F_{o} \mathcal{K}\right)$, that is, $F_{o} \mathcal{S} E_{o}$ is transitive. By [4], this implies that $F_{o} \mathcal{S} E_{o}$ is $\mathrm{w}^{*}$-dense in $\mathcal{B}\left(E_{o} \mathcal{H}, F_{o} \mathcal{K}\right)$. Since it is norm-closed, it must contain all rank one operators, and thus $F_{o} T E_{o}$ must be a finite sum of rank one operators in $F_{o} \mathcal{S} E_{o}$.

Remark 5.1 In Theorem 5.1,

(i) The assumption that $\mathcal{S}$ is norm closed cannot be omitted. Indeed Arveson [4] constructed a $\mathrm{w}^{*}$-closed masa bimodule $\mathcal{M}$ which is not reflexive; 
hence there exists a trace class operator $T$ which annihilates $\mathcal{M}$ but not $\operatorname{Ref}(\mathcal{M})$. Let $\mathcal{S}={ }^{\perp} \mathcal{M}$. All rank one operators in $\mathcal{S}$ annihilate $\operatorname{Ref}(\mathcal{M})$, so $T$ cannot be in their trace-norm closed linear span.

(ii) The assumption that $\operatorname{rank} T<\infty$ cannot be omitted: the conclusion may fail even when $T$ is trace class and $\mathcal{S}$ is a strongly reflexive bimodule (see section 10).

Both these assertions use the concept of spectral synthesis (see section 9).

In view of the remarks of the previous section, Theorem 5.1 immediately gives

Theorem 5.2 Every strongly reflexive masa bimodule $\mathcal{S}$ is the weak operator closure of its rank one subspace.

Proof Let $\mathcal{R}=\mathcal{R}_{1}(\mathcal{S})$. By Proposition 4.1, we need to prove that $\mathcal{F}\left(\mathcal{R}^{\perp}\right) \subseteq$ ${\overline{\mathcal{R}_{1}\left(\mathcal{R}^{\perp}\right)}}^{1}$. Since $\mathcal{R}^{\perp}$ is a norm-closed masa bimodule, this follows from Theorem 5.1.

Let me emphasise once again that, contrary to the unital algebra case [20], the result may fail for the $\mathrm{w}^{*}$-topology (see section 10 ).

To summarise:

- Any WOT-closed masa bimodule which is WOT-generated by rank one operators is reflexive (hence strongly reflexive).

- There exists a $\mathrm{w}^{*}$-closed masa bimodule which is $\mathrm{w}^{*}$-generated by rank one operators and is not reflexive (but its WOT-closure is).

However,

- Any $\mathrm{w}^{*}$-closed unital algebra containing a masa, which is $\mathrm{w}^{*}$-generated by rank one operators, is reflexive (Laurie-Longstaff [20]).

\section{The case of matrix units}

To motivate the notion of supporting set and the techniques that use it, consider the easy case of bimodules over discrete masas:

Let $\mathcal{R} \subseteq \mathcal{B}(\mathcal{H}, \mathcal{K})$ be a set of matrix units with respect to given orthonormal bases $\left\{e_{j}\right\}$ of $\mathcal{H}$ and $\left\{f_{i}\right\}$ of $\mathcal{K}$. Thus there exists a subset $\kappa \subseteq \mathbb{N} \times \mathbb{N}$ such 
that

$$
\mathcal{R}=\left\{f_{j} \otimes e_{i}^{*}:(i, j) \in \kappa\right\} .
$$

Let $\mathcal{S}=\overline{[\mathcal{R}]}^{w *}$. If we set $\mathcal{R}_{\vee}=\left\{e_{i} \otimes f_{j}^{*}:(i, j) \notin \kappa\right\}$, it is not hard to show that $\mathcal{S}=\mathcal{R}_{\vee}^{\perp}$. By Theorem 2.1, this yields

Proposition 6.1 If $\mathcal{S}=\overline{[\mathcal{R}}^{w *}$, then $\mathcal{S}$ is reflexive (hence strongly reflexive).

Note that in fact $\mathcal{S}$ consists of all $T \in \mathcal{B}(\mathcal{H}, \mathcal{K})$ which are supported in $\kappa$ in the sense that $(n, m) \notin \kappa \Rightarrow\left\langle T e_{n}, f_{m}\right\rangle=0$.

The proof of Proposition 6.1 uses the existence of a bounded approximate identity in the rank one subalgebras of the diagonal masas $\mathcal{A}=\left\{\left[e_{i}\right]\right\}^{\prime \prime}$ and $\mathcal{B}=\left\{\left[f_{j}\right]\right\}^{\prime \prime}$. This method in fact yields more:

Proposition 6.2 Any $w^{*}$-closed $(\mathcal{B}, \mathcal{A})$-bimodule $\mathcal{S}$ is generated in the $w^{*}$ topology by its rank one subspace, and hence is strongly reflexive.

It is clear that this may fail if the masas are not discrete; the fact that the previous proposition may also fail is rather deeper (section 10).

These ideas are also in the motivation of the work of Muhly, Saito and Solel [23]. Very briefly, given a von Neumann algebra $\mathcal{U}$ containing a Cartan subalgebra $\mathcal{A}$ they prove that any $\mathrm{w}^{*}$-closed $\mathcal{A}$-bimodule $\mathcal{M} \subseteq \mathcal{U}$ can be 'represented' as the set of all 'matrices' supported on a suitable set (the multiplication may have to be twisted by a cocycle). A Cartan subalgebra of $\mathcal{U}$ is a masa (relative to $\mathcal{U}$ ) with additional properties, which need not concern us here; in case $\mathcal{U}=\mathcal{B}(\mathcal{H})$, the only Cartan subalgebras are the discrete masas.

However, their notion of 'representation' is (isometric) isomorphism, not unitary equivalence. They first represent $\mathcal{U}$ on a Hilbert space $\mathcal{K}$ (up to *-isomorphism) so that it acquires a separating vector. Then all $\mathrm{w}^{*}$-closed subspaces of $\mathcal{U}$ are automatically reflexive as subspaces of $\mathcal{B}(\mathcal{K})$ by a result of Loginov-Shulman [21]. We have seen (Remark 5.1 (i)) that not all w*closed masa bimodules are reflexive in the Hilbert space where they 'live'.

These remarks show that, in order to deal with spatial representations of bimodules over arbitrary masas, we will have to adopt a different, 'nondiscrete', approach. Our 'building blocks' will be rank one operators, not in the bimodule itself, but in its annihilator. 


\section{Supports}

Given masas $\mathcal{A} \subseteq \mathcal{B}(\mathcal{H})$ and $\mathcal{B} \subseteq \mathcal{B}(\mathcal{K})$, we wish to 'choose co-ordinates' in order to represent (up to unitary equivalence) all reflexive $(\mathcal{B}, \mathcal{A}$ )-bimodules simultaneously in a 'coherent way'. As is well known, $\mathcal{H}$ and $\mathcal{K}$ can be represented as $L^{2}$ spaces so that $\mathcal{A}$ and $\mathcal{B}$ correspond to the multiplication masas.

Thus in the sequel we assume that $\mathcal{H}=L^{2}(X, \mu), \mathcal{K}=L^{2}(Y, \nu)$ and that $\mathcal{A} \subseteq \mathcal{B}(\mathcal{H})$ and $\mathcal{B} \subseteq \mathcal{B}(\mathcal{K})$ are the corresponding multiplication masas. If $\alpha$ is a Borel subset of $X$ (resp. $\beta$ is a Borel subset of $Y$ ) we write $E(\alpha) \in \mathcal{A}$ (resp. $F(\beta) \in \mathcal{B}$ ) for the corresponding projections.

Definition 7.1 Let $T \in \mathcal{B}(\mathcal{H}, \mathcal{K})$ and let $\kappa \subseteq X \times Y$ be any set. We say that $\kappa$ supports $T$ if, for any Borel rectangle $\alpha \times \beta$,

$$
(\alpha \times \beta) \cap \kappa=\emptyset \Rightarrow F(\beta) T E(\alpha)=0 .
$$

A subset $\mathcal{S} \subseteq \mathcal{B}(\mathcal{H}, \mathcal{K})$ is supported in $\kappa$ if all its operators are supported in $\kappa$.

Note that if a set $\kappa$ supports $\mathcal{S}$ then it supports $\operatorname{Ref}(\mathcal{S})$. Also, this notion clearly reduces to the one introduced in section 6 for the case of discrete masas.

Definition 7.2 Let $\kappa \subseteq X \times Y$ be any set. Define

$$
\mathcal{M}_{\max }(\kappa)=\{T \in \mathcal{B}(\mathcal{H}, \mathcal{K}): \kappa \text { supports } T\} .
$$

It is clear that $\mathcal{M}_{\max }(\kappa)$ is a $\mathrm{w}^{*}$-closed $(\mathcal{B}, \mathcal{A})$-bimodule.

Proposition 7.1 Any $\mathcal{M}_{\max }(\kappa)$ is a reflexive $(\mathcal{B}, \mathcal{A})$-bimodule.

The idea of the proof is to show that $\mathcal{M}_{\max }(\kappa)=\mathcal{R}^{\perp}$ where

$$
\mathcal{R}=\{E(\alpha) R F(\beta) \in \mathcal{B}(\mathcal{K}, \mathcal{H}): \operatorname{rank} R=1,(\alpha \times \beta) \cap \kappa=\emptyset\} .
$$

In the converse direction we have

Theorem 7.2 If $\mathcal{M} \subseteq \mathcal{B}(\mathcal{H}, \mathcal{K})$ is a reflexive $(\mathcal{B}, \mathcal{A})$-bimodule, there exists a set $\kappa_{M} \subseteq X \times Y$ such that $\mathcal{M}_{\max }\left(\kappa_{M}\right)=\mathcal{M}$. 
For the proof, see [9].

To what extent is the set $\kappa_{M}$ unique?

Recall that a unital algebra $\mathcal{A}$ is reflexive if and only if there is a set $\mathcal{P}$ of subspaces such that $\mathcal{A}=\operatorname{Alg} \mathcal{P}$. The set $\mathcal{P}$ is highly non-unique. But there is only one reflexive lattice $\mathcal{L}$ with $\mathcal{A}=\operatorname{Alg} \mathcal{L}$, namely $\mathcal{L}=$ Lat $\mathcal{A}$.

The notion corresponding to reflexivity for sets is $\omega$-closure. To introduce this, first note that a set $\kappa \subseteq X \times Y$ can be modified by 'negligible' sets without affecting $\mathcal{M}_{\max }(\kappa)$. More precisely,

Remark 7.1 If $\kappa, \sigma$ are subsets of $X \times Y$ and $\kappa \Delta \sigma \subseteq \chi \times Y \cup X \times \psi$ where $\mu(\chi)=0=\nu(\psi)$ (we then say that $\kappa \Delta \sigma$ is marginally null and write $\kappa \Delta \sigma \stackrel{m}{\cong} \emptyset)$ then $\mathcal{M}_{\max }(\kappa)=\mathcal{M}_{\max }(\sigma)$.

The converse of this holds when the sets are sufficiently 'well behaved':

Definition 7.3 A set $\kappa \subseteq X \times Y$ is said to be $\omega$-open if $\kappa \stackrel{m}{\cong} \cup_{n}\left(\alpha_{n} \times\right.$ $\left.\beta_{n}\right)$ where $\alpha_{n} \subseteq X$ and $\bar{\beta}_{n} \subseteq Y$ are Borel sets. A set is $\omega$-closed if its complement is $\omega$-open.

Theorem 7.3 (uniqueness) If $\mathcal{M}_{\max }(\kappa)=\mathcal{M}_{\max }(\sigma)$ and both $\kappa, \sigma$ are $\omega$-closed then $\kappa \stackrel{m}{\cong} \sigma$.

Observe that if a set $\kappa \subseteq X \times Y$ is marginally null, then it can support no (nonzero) operators. The crucial step in the proof of the uniqueness theorem is a partial converse to this:

Arveson's null set Theorem [4] If the complement of $\kappa$ is a countable union of Borel rectangles, and it supports no operators, then it is marginally null.

Definition 7.4 (a) Given a set $\mathcal{S} \subseteq \mathcal{B}(\mathcal{H}, \mathcal{K})$, let $\mathcal{M}=\operatorname{Ref}(\mathcal{B S} \mathcal{A})$. The set $\kappa_{M}$ constructed in Theorem 7.2 is called the $\omega$-support of $\mathcal{S}$ and denoted $\operatorname{supp}_{\omega}(\mathcal{S})$.

(b) The $\omega$-closure $\mathrm{cl}_{\omega}(\kappa)$ of a set $\kappa \subseteq X \times Y$ is defined to be $\operatorname{supp}_{\omega}\left(\mathcal{M}_{\max }(\kappa)\right)$.

Thus $\omega$-supports and $\omega$-closures are defined up to marginally null sets. Note that $\omega$-closure is not a topological closure operation. 
Remark 7.2 It can be easily shown that $\mathrm{cl}_{\omega}(\kappa)$ contains $\kappa$ up to marginally null sets. The uniqueness theorem shows that an $\omega$-closed set $\kappa$ is 'reflexive' in the sense that if $\mathcal{M}=\mathcal{M}_{\max }(\kappa)$ then $\kappa \stackrel{m}{\cong} \operatorname{supp}_{\omega}\left(\mathcal{M}_{\max }(\kappa)\right)$ (recall that a set $\mathcal{L}$ of subspaces is called reflexive when $\mathcal{L}=\operatorname{Lat} \operatorname{Alg} \mathcal{L})$.

Remark 7.3 Given a reflexive $(\mathcal{B}, \mathcal{A})$-bimodule $\mathcal{M} \subseteq \mathcal{B}(\mathcal{H}, \mathcal{K})$, we may find compact metric spaces $X, Y$ such that $\mathcal{A}, \mathcal{B}$ are unitarily equivalent to the multiplication masas acting on the corresponding $L^{2}$ spaces and $\mathcal{M}$ is unitarily equivalent to $\mathcal{M}_{\max }(\kappa)$ where $\kappa$ is a closed set. This result is an extension of the traditional approach, due essentially to Arveson [4]. However the topologies depend on $\mathcal{M}$, not only on the masas.

By contrast, in our approach, once a spatial representation is fixed for the masas $\mathcal{A}$ and $\mathcal{B}$, all $(\mathcal{B}, \mathcal{A})$-bimodules can be "simultaneously" written as $\mathcal{M}_{\max }(\kappa)$ for $(\omega$-closed) sets $\kappa$.

Remark 7.4 There is a close connection between reflexive masa bimodules and reflexive algebras containing masas. This is provided by Arveson's ' $2 \times 2$ matrix trick': given an $(\mathcal{B}, \mathcal{A})$-bimodule $\mathcal{M} \subseteq \mathcal{B}(\mathcal{H}, \mathcal{K})$, the set

$$
\mathcal{U}=\left(\begin{array}{cc}
\mathcal{B} & \mathcal{M} \\
0 & \mathcal{A}
\end{array}\right)
$$

is easily seen to be a (unital) algebra containing a masa. It can be shown that $\mathcal{U}$ is reflexive as a unital algebra if and only if $\mathcal{M}$ is reflexive as a subspace. However strong reflexivity of $\mathcal{M}$ does not imply that $\mathcal{U}$ is strongly reflexive.

\section{Strongly reflexive masa bimodules}

The notion of $\omega$-support allows a particularly transparent characterisation of these bimodules. They are precisely the masa bimodules whose $\omega$-supports are the $\omega$-closures of $\omega$-open sets:

Theorem 8.1 A subset $\mathcal{M} \subseteq \mathcal{B}(\mathcal{H}, \mathcal{K})$ is a strongly reflexive $(\mathcal{B}, \mathcal{A})$-bimodule if and only if there exists a countable family $\left\{\gamma_{n} \times \delta_{n}\right\}$ of Borel rectangles in $X \times Y$ such that

$$
\mathcal{M}=\mathcal{M}_{\max }\left(\cup_{n}\left(\gamma_{n} \times \delta_{n}\right)\right)
$$

Remark 8.1 In the situation of Theorem 8.1, compact metric topologies can be chosen (depending on $\mathcal{M}$ as well as $\mathcal{A}, \mathcal{B}$ ) so that $\mathcal{M}$ is unitarily 
equivalent to $\mathcal{M}_{\max }(\kappa)$ where $\kappa$ is the closure of an open set. The converse, however, is not always true.

\section{Spectral synthesis}

Many interesting examples of CSL algebras and, more generally, masa bimodules, come from the interplay between operator theory and harmonic analysis. We briefly recall the few facts that we shall need.

The Fourier-Wiener algebra is

$$
A(\mathbb{T})=\left\{f \in C(\mathbb{T}): \sum_{n \in \mathbb{Z}}|\widehat{f}(n)|<\infty\right\}
$$

This is isomorphic to $\ell^{1}(\mathbb{Z})$ via the Fourier transform $f \rightarrow \widehat{f}$. Thus $A(\mathbb{T})$ consists of all functions on the circle with absolutely convergent Fourier series and is a Banach space under the norm

$$
\|f\|_{A}=\sum_{n \in \mathbb{Z}}|\widehat{f}(n)| \geq\|f\|_{\infty} .
$$

The space $P M(\mathbb{T})$ of pseudomeasures is the dual of $\left(A(\mathbb{T}),\|\cdot\|_{A}\right)$. It is isometrically isomorphic to $\ell^{\infty}(\mathbb{Z})$ via the Fourier transform; specifically for $\phi \in P M(\mathbb{T})$ we define

$$
\widehat{\phi}(n)=\phi\left(e_{-n}\right)
$$

where $e_{n}(t)=\exp ($ int $)$, and we have

$$
\phi(f)=\sum_{n} \widehat{\phi}(-n) \widehat{f}(n)
$$

for $\phi \in P M(\mathbb{T})$ and $f \in A(\mathbb{T})$.

We represent $P M(\mathbb{T})$ and $A(\mathbb{T})$ as operators on $L^{2}(\mathbb{T})$ so as to preserve the duality (8): For $\phi \in P M(\mathbb{T})$ and $f \in A(\mathbb{T})$ we define

$$
C_{\phi}\left(e_{n}\right)=\widehat{\phi}(-n) e_{n} \text { and } T_{f}\left(e_{n}\right)=\widehat{f}(n) e_{n} .
$$

Then $C_{\phi} \in \mathcal{B}\left(L^{2}(\mathbb{T})\right), T_{f} \in \mathcal{C}_{1}\left(L^{2}(\mathbb{T})\right)$ and $\left\|C_{\phi}\right\|=\|\phi\|_{A^{*}},\left\|T_{f}\right\|_{1}=\|f\|_{A}$. It follows that

$$
\operatorname{tr}\left(C_{\phi} T_{f}\right)=\phi(f) .
$$

Definition 9.1 Let $E \subseteq \mathbb{T}$ be a closed set. A pseudomeasure $\phi$ is said to be supported in $E$ if $\phi(f)=0$ for any $f \in A(\mathbb{T})$ with $\operatorname{supp}(f) \cap E=\emptyset$. 
Note that such an $f$ vanishes in a neighbourhood of $E$. Also note that a point measure $\delta_{t}$ is supported in $E$ if and only if $t \in E$.

Definition 9.2 A closed set $E \subseteq \mathbb{T}$ is said to satisfy spectral synthesis if any $\phi \in P M(\mathbb{T})$ which is supported in $E$ can be 'synthesised' by point measures supported in $E$, that is

$$
\phi \in{\overline{\left[\delta_{t}: t \in E\right]}}^{w *} .
$$

Thus if $E \subseteq \mathbb{T}$ fails spectral synthesis, there exists $\phi \in P M(\mathbb{T})$ which is supported in $E$ and $f \in A(\mathbb{T})$ which vanishes on $E$ such that $\phi(f) \neq 0$.

\section{0. $\mathrm{w}^{*}$ density of the rank one subspace}

We are now in a position to construct a strongly reflexive masa bimodule $\mathcal{M}$ such that ${\overline{\mathcal{R}_{1}(\mathcal{M})}}^{w *} \neq \mathcal{M}$. This will be described in the form $\mathcal{M}_{\max }\left(\kappa_{E}\right)$ where

$$
\kappa_{E}=\{(t, s): t-s \in E\}
$$

for a suitable subset $E$ of the circle $\mathbb{T}$. We will need two lemmas.

Lemma 10.1 (Froelich [10]) A pseudomeasure $\phi$ is supported in a closed set $E \subseteq \mathbb{T}$ if and only if the corresponding operator $C_{\phi}$ is supported in $\kappa_{E}$, that is $C_{\phi} \in \mathcal{M}_{\max }\left(\kappa_{E}\right)$.

Lemma 10.2 Let $f \in A(\mathbb{T})$ with $f \mid E=0$. Then $T_{f} \perp \mathcal{R}_{1}\left(\mathcal{M}_{\max }\left(\kappa_{E}\right)\right)$.

Proof Suppose $h \otimes g^{*}$ is supported in $\kappa_{E}$. Then

$$
\begin{aligned}
\operatorname{tr}\left(T_{f}\left(h \otimes g^{*}\right)\right) & =\left\langle T_{f} h, g\right\rangle=\sum_{n} \widehat{f}(n) \widehat{h}(n) \overline{\widehat{g}(n)} \\
& =\sum_{n} \widehat{f * h}(n) \overline{\widehat{g}(n)} \\
& =\int(f * h)(t) \overline{g(t)} d t \\
& =\int\left(\int f(s-t) h(s) d s\right) \overline{g(t)} d t \\
& =\iint f(s-t) h(s) \overline{g(t)} d s d t
\end{aligned}
$$


which vanishes, because $f(s-t)=0$ when $(s, t) \in \kappa_{E}$ while $h(s) \overline{g(t)}=0$ for almost all $(s, t) \notin \kappa_{E}$.

Now let $E \subseteq \mathbb{T}$ be a closed set failing spectral synthesis. Fix $\phi \in P M(\mathbb{T})$ supported in $E$ and $f \in A(\mathbb{T})$ with $f \mid E=0$ such that $\phi(f) \neq 0$. If $\mathcal{M}=$ $\mathcal{M}_{\text {max }}\left(\kappa_{E}\right)$, then $T_{f}$ annihilates $\mathcal{R}_{1}(\mathcal{M})$ but not $\mathcal{M}$ because $\operatorname{tr}\left(C_{\phi} T_{f}\right)=$ $\phi(f) \neq 0$ while $C_{\phi} \in \mathcal{M}$. It remains to find such a set $E$ for which $\mathcal{M}_{\max }\left(\kappa_{E}\right)$ is strongly reflexive.

Lemma 10.3 ([9]) If $E \subseteq \mathbb{T}$ is the closure of its interior, then $\mathcal{M}_{\max }\left(\kappa_{E}\right)$ is strongly reflexive.

Lemma 10.4 (Colela [5]) There exists a closed set $E \subseteq \mathbb{T}$ failing spectral synthesis which is the closure of its interior.

We have shown

Theorem 10.5 ([9]) There exists a strongly reflexive masa bimodule $\mathcal{M}$ for which rank-one density fails in the $w^{*}$-topology.

This also yields a strongly reflexive masa bimodule $\mathcal{S}$ and a trace-class operator $T \in \mathcal{S}$ which is not in the trace-norm closure of $\mathcal{R}_{1}(\mathcal{S})$. Indeed let $\mathcal{S}=\mathcal{R}_{1}(\mathcal{M})^{\perp}$. Then there exists $T \in \mathcal{C}_{1} \cap \mathcal{S}$ not annihilating the whole of $\mathcal{M}$. But, since $\mathcal{M}$ is strongly reflexive, all rank one operators in $\mathcal{S}$ annihilate $\mathcal{M}$ (lemma 3.1), hence $T \notin{\overline{\mathcal{R}_{1}(\mathcal{S})}}^{1}$.

\section{References}

1. Anoussis, M. , Katavolos, A. and Lambrou, M.S (1993) On the reflexive algebra with two invariant subspaces, J. Operator Theory 30, 267-299.

2. Argyros, S. , Lambrou, M.S. and Longstaff, W.E.(1991) Atomic Boolean subspace lattices and applications to the theory of bases, Mem. Amer. Math. Soc. 445, Providence, RI.

3. Arveson, W. (1967) Analyticity in operator algebras, Amer. J. Math. 89, 578-642.

4. Arveson, W. (1974) Operator Algebras and Invariant subspaces, Ann. Math. (2) 100, 433-532.

5. Colela, D. (1983) On spectral synthesis for sets of the form $E=\operatorname{cl}(\operatorname{int}(E))$, Proc. Amer. Math. Soc. 89, 236-238.

6. Davidson, K.R. (1988) Nest Algebras, Pitman research notes in Mathematics 191, Longman, UK.

7. Erdos, J.A. (1968) Operators of finite rank in nest algebras, J. London Math. Soc. 43, 391-397.

8. Erdos, J.A. (1986) Reflexivity for subspace maps and linear spaces of operators, Proc. London Math. Soc. (3) 52, 582-600.

9. Erdos, J.A., Katavolos, A. and Shulman, V.S. (preprint) Rank one subspaces of bimodules over maximal abelian selfadjoint algebras. 
10. Froelich, J. (1988) Compact operators, invariant subspaces and spectral synthesis, J. Funct. Anal. 81 No. 1, 1-37.

11. Gilfeather, F, and Larson, D.R. (1982) Structure in reflexive subspace lattices, J. London Math. Soc. (2) 26, 117-131.

12. Halmos, P.R (1971) Reflexive lattices of subspaces, J. London Math. Soc. (2) 4, 257-263.

13. Hopenwasser, A. and Moore, R. (1983) Finite rank operators in reflexive operator algebras, J. London Math. Soc. (2) 27, 331-338.

14. Trent, T., quoted in Hopenwasser, A., Laurie, C. and Moore, R.(1984) Reflexive algebras with completely distributive subspace lattices, J. Operator Theory 11, 91108.

15. Kraus, J. and Larson D.R. (1985) Some applications of a technique for constructing reflexive operator algebras, J. Operator Theory 13, 227-236.

16. Kadison, R.V. and Singer, I.M. (1960) Triangular operator algebras, Amer. J. Math 82, 227-259.

17. Lambrou, M.S. and Longstaff, W.E. (1992) Finite rank operators leaving double triangles invariant, J. London Math. Soc. (2) 45, 153-168.

18. Larson, D.R. (1982) Annihilators of operator algebras, Topics in Modern Operator Theory 6, pp. 119-130, Birkhauser verlag, Basel.

19. Larson, D.R and Wogen, W.R. (1990) Reflexivity properties of $T \oplus 0$, J. Funct. Anal.92 No. 2, 448-467.

20. Laurie, C. and Longstaff, W. (1983) A note on rank one operators in reflexive algebras, Proc. Amer. Math. Soc. 89, 293-297.

21. Loginov, A.I. and Shulman, V.S. (1975) Hereditary and intermediate reflexivity of W*-algebras, (Russian) Izv. Akad. Nauk. SSSR Ser. Mat. 39 (1975), 1260-1273; (English transl.) Math. USSR Izv. 9 1189-1201.

22. Longstaff, W.(1975) Strongly reflexive lattices, J. London Math. Soc. (2) 11, 491498.

23. Muhly, P.S., Saito, K-S. and Solel, B. (1988) Coordinates for triangular operator algebras, Ann. Math. 127, 245-278.

24. Papadakis, M.(1993), Non-selfadjoint operator algebras on Hilbert spaces, Ph.D. Thesis, University of Athens (in Greek).

25. Ringrose, J.R. (1965) On some algebras of operators, Proc. London Math. Soc. (3) 15, $61-83$. 